http://www.jfas.info

\title{
MODELING AND CONTROLLER DESIGN ON ARX MODEL OF ELECTRO-HYDRAULIC ACTUATOR
}

\author{
N. Ishak ${ }^{*}$, N. S. Hamdan, M. Tajuddin and R. Adnan \\ Frontier Materials and Industry Application, UiTM-RMI-CoRe FMIA \\ Faculty of Electrical Engineering, Universiti Teknologi MARA, 40450 Shah Alam, Selangor, \\ Malaysia
}

Published online: 05 October 2017

\begin{abstract}
Electro-hydraulic actuator (EHA) is commonly used in industry for its linear movement, quick response and accurate positioning of heavy loads. However, the uncertainties, highly nonlinearities and time varying characteristic of EHA caused difficulties in controlling the system. This paper studies the performance of Fuzzy PID controller on ARX model parameters of vertical position of electro-hydraulic actuator. The system transfer function is obtained via system identification technique using MATLAB Toolbox. The performance of the controller is analyzed through simulation by using step and square type reference input. The roots mean squared error show that the controllers with obtained model of 50ms sampling time give better performance.
\end{abstract}

Keywords: EHA; Fuzzy PID; ARX.

Author Correspondence, e-mail: norlelaishak@salam.uitm.edu.my doi: http://dx.doi.org/10.4314/jfas.v9i4s.9 


\section{INTRODUCTION}

Electro-Hydraulic Actuator (EHA) has been commonly used in industry for its linear movement, fast response and accurate positioning of heavy load making it important tools for industrial processes. The advantages of EHA over drive system are high power to weight ratio, smooth response, accurate positioning of heavy load and good power capability [1]. In addition, it improvises the safety and reliability aspect and also simplifies system architecture. Based on the characteristics, it is widely used in automotive and aerospace industries. However, it also has its own behavior such as high nonlinearities, uncertainties and time-varying characteristics that will cause difficulties in controlling process [3]. There are factors that will influence some of the properties such as viscosity and temperature of the fluid. Thus advanced system identification is necessary to control the EHA system to achieve good performance of the system.

In order to design a controller that control the actuator, a model that shows the system's behavior first need to be obtained by using system identification method. System identification is the proses of obtaining system model through system's input-output data (stimulus-response) [2]. This method is much easier than Physical law method because derivation of mathematical model is needed for modeling [4]. The input-output data was then used in MATLAB System Identification Toolbox to obtain the model transfer function.

The major role of the controller is to find the set of command that fit to the system to reach the desired state with minimum deviation. The most commonly used controller in control system design is PID controller for its simplicity and has well understood features [5]. The implementations of PID controller need convenient values for $\mathrm{K}_{\mathrm{p}}, \mathrm{K}_{\mathrm{i}}$ and $\mathrm{K}_{\mathrm{d}}$ shown in Equation

$$
\mathrm{u}(\mathrm{k})=\mathrm{K}_{\mathrm{p}} \mathrm{e}(\mathrm{k})+\sum_{\mathrm{i}=0}^{\mathrm{k}} \mathrm{e}(\mathrm{i})+\mathrm{K}_{\mathrm{d}}[\mathrm{e}(\mathrm{k})-\mathrm{e}(\mathrm{k}-1)]
$$

Over the good performance, the PID method poorly control position in hydraulic system as it does not fit for controlling system with large amount of lag, parameter variations and uncertainty in models. Further research and studies has been conducted to improve PID control performances by developing Fuzzy Logic Control technique. Recently, fuzzy logic control has 
been applied to improve the robustness and hybrid control of fuzzy and PID. In the meantime, Fuzzy PID controllers are used in many applications such as speed control, robot manipulator and DC motor [6-9].

This paper discusses the methodology to obtain the model of EHA using MATLAB System Identification Toolbox. The transfer function obtain from model identification is then applied to the Fuzzy PID controller. Simulations are conducted in MATLAB Simulink to observe the performance of the proposed controller. The results using two different inputs and four model of EHA with different sampling times will be compared and discussed.

\section{METHODOLOGY}

\subsection{Plant}

The hardware setup is shown as in Fig. 1. The single ended cylinder of EHA consists of bidirectional cylinder with $25 \mathrm{~mm}$ rod size; $40 \mathrm{~mm}$ bore size and $150 \mathrm{~mm}$ stroke length. There is displacement sensor on the upper part of the cylinder rod. In the meantime, electronic control valve control the pressure of the fluid of electro-hydraulic actuator. There are proportional and directional types of this control valve. The current used is in the range of 4-20 $\mathrm{mA}$ while the valve input voltage is $\pm 10 \mathrm{~V} \mathrm{dc}$.

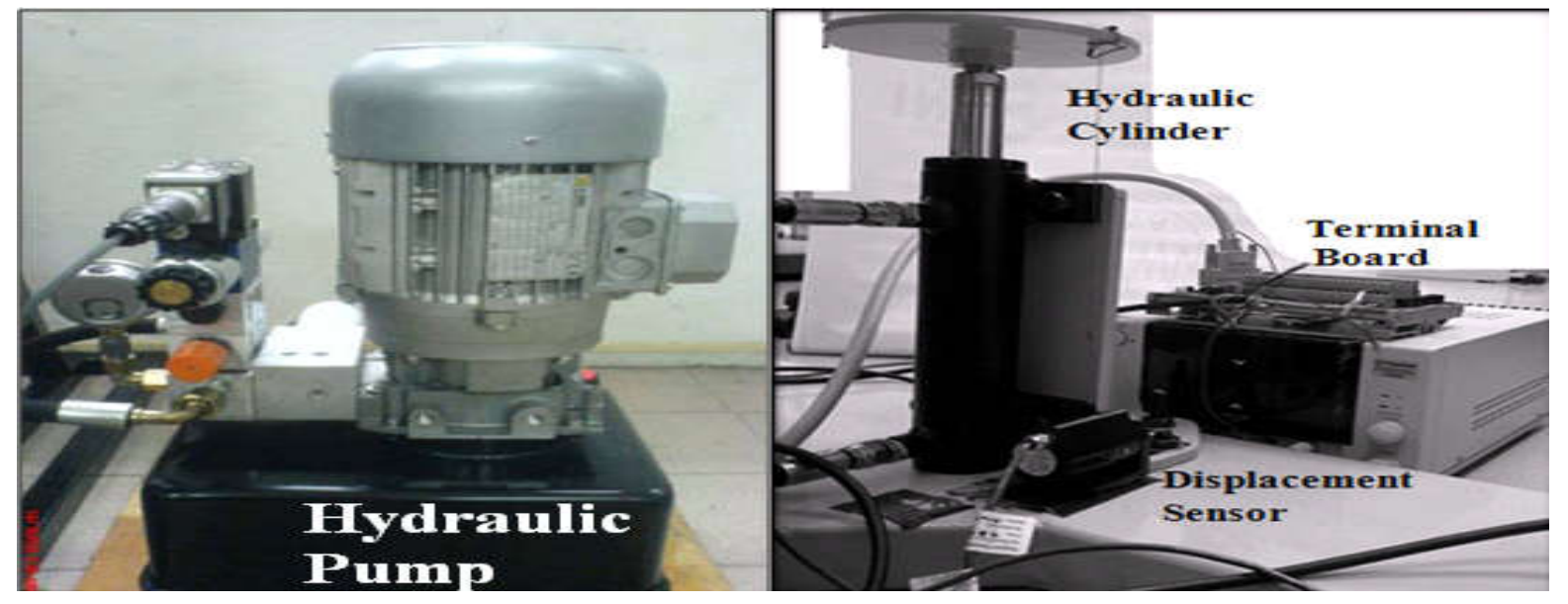

Fig.1. Electro-Hydraulic Actuator (EHA)

\subsection{Model Identification}

The input test signal use can be generated using Equation (2). For this work, the input test signal used is represented by Equation (3) with three different values of frequencies [10]. The generated input test signal is shown in Fig. 2. 
$u(k)=\sum_{i=1}^{p} a_{i} \cos \omega_{i} t_{s} k$

where $a_{i}$ : amplitude, $\omega_{i}$ : frequency and $t_{s}:$ sampling-time $(\mathrm{sec})$.

$v_{\text {in }}(k)=2 \cos 0.5 t_{s} k+2 \cos 0.7 t_{s} k+\cos t_{s} k$

Higher model may produce unstable output signal. Thus, the model obtained limited to second and third order only. For this studies, third order model ARX331 was selected as the best model to represent the nearest model of true plant. The input-output signals are shown in Fig.

2.
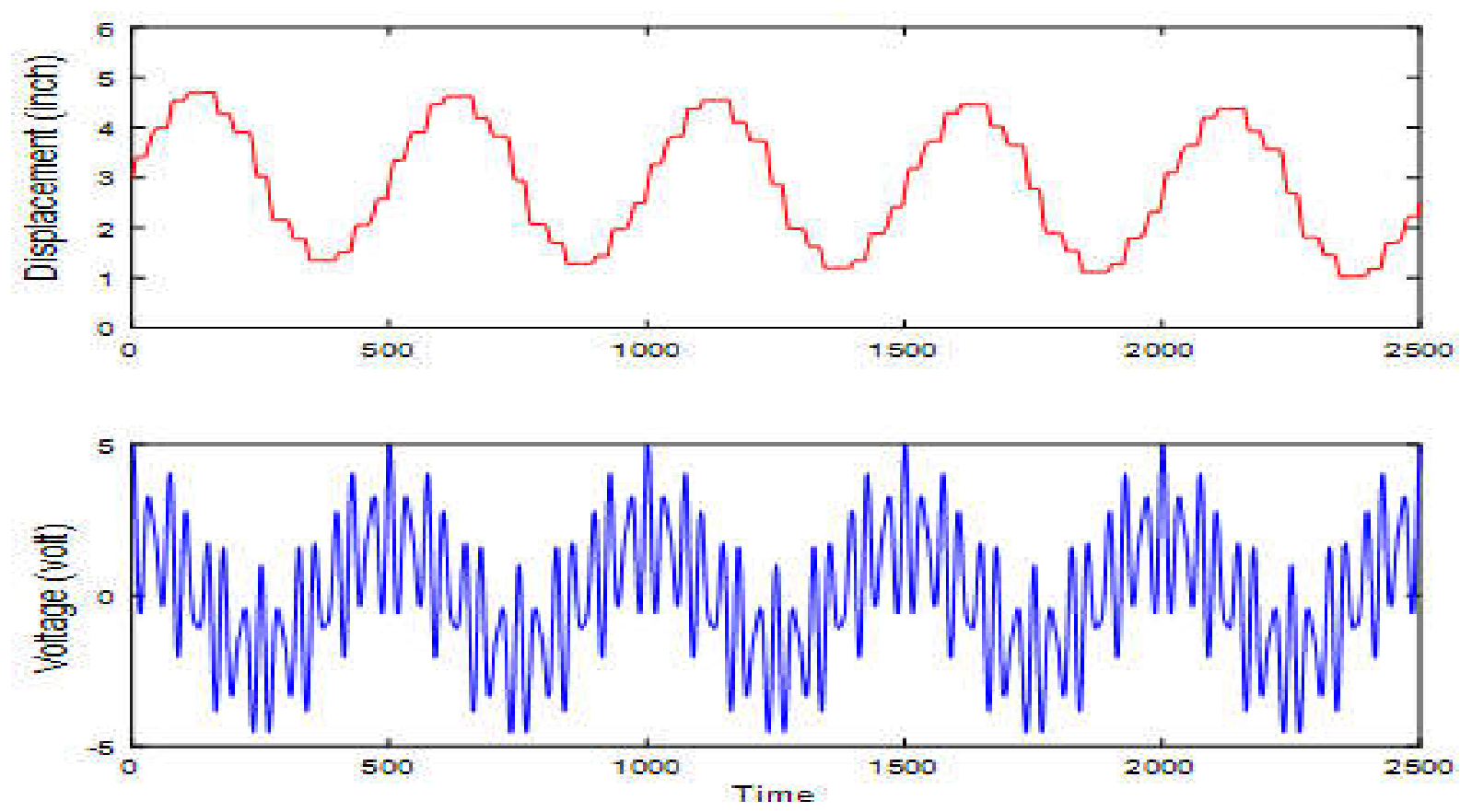

Fig.2. Input and output signal for 50ms sampling time

Using the collected data and MATLAB system identification toolbox, two plant models with different zero positions were obtained for simulation studies purposes. One model having one zero outside and far away from the unity circle and known as Model 1. Another one having one zero outside and near to the unity circle and known as Model 2. Model 1 using 40ms sampling-time and Model 2 using 50ms sampling-time. Another two plant models with different zero positions were also obtained for simulation studies purposes. One model was having all zeros inside and far away from the unity circle and known as Model 3. Another one was having all zeros inside the unity circle, but close to unity circle and known as Model 4. Model 3 using 60ms sampling-time and Model 4 using 65ms sampling-time. Their pole-zero plots are given in Fig. 3. 

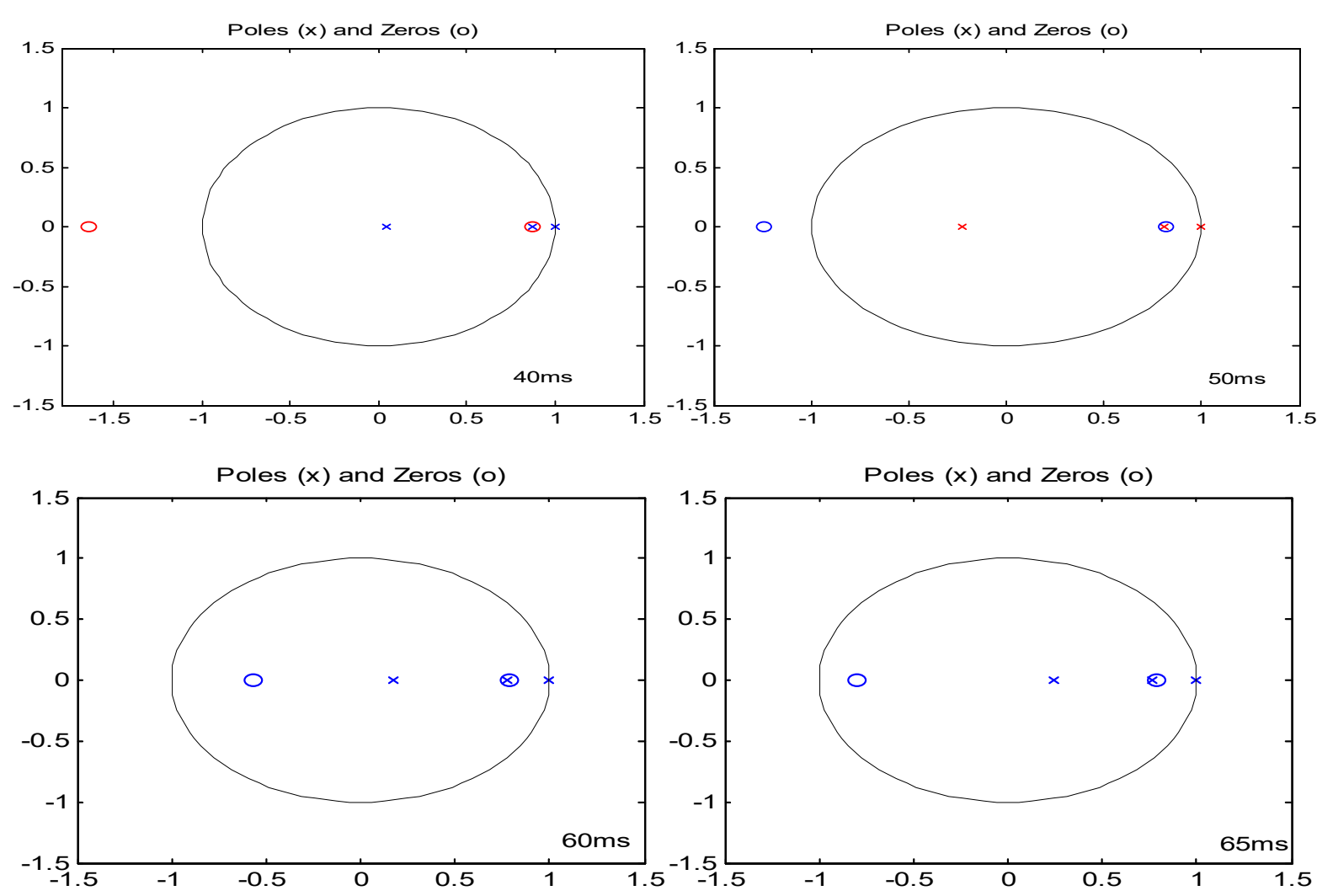

Fig.3. Pole-zero plot

Table 1 shown the obtain four model of ARX331 with different sampling time.

Table 1. ARX331 model of EHA system

\begin{tabular}{ccc}
\hline Sampling Time & Best Fit (\%) & Transfer Function \\
\hline $40 \mathrm{~ms}$ & 89.96 & $\frac{B_{o}\left(z^{-1}\right)}{A_{o}\left(z^{-1}\right)}=\frac{0.0039 z^{-1}+0.0029 z^{-2}-0.0055 z^{-3}}{1-1.9070 z^{-1}+0.9414 z^{-2}-0.0341 z^{-3}}$ \\
$50 \mathrm{~ms}$ & 89.86 & $\frac{B_{o}\left(z^{-1}\right)}{A_{o}\left(z^{-1}\right)}=\frac{0.0087 z^{-1}+0.0037 z^{-2}-0.0088 z^{-3}}{1-1.580 z^{-1}+0.3938 z^{-2}+0.1861 z^{-3}}$ \\
$60 \mathrm{~ms}$ & 90.42 & $\frac{B_{o}\left(z^{-1}\right)}{A_{o}\left(z^{-1}\right)}=\frac{0.0072 z^{-1}-0.0018 z^{-2}-0.0031 z^{-3}}{1-1.956 z^{-1}+1.095 z^{-2}-0.1384 z^{-3}}$ \\
$65 \mathrm{~ms}$ & 90.67 & $\frac{B_{o}\left(z^{-1}\right)}{A_{o}\left(z^{-1}\right)}=\frac{0.0061 z^{-1}+0.0004 z^{-2}-0.0038 z^{-3}}{1-2.002 z^{-1}+1.184 z^{-2}-0.1817 z^{-3}}$
\end{tabular}

\subsection{Controller Design}

This section discussed the development of the fuzzy PID controller to control the position of electro-hydraulic actuator (EHA). Fuzzy PID has the same linear structure as conventional PID, constant coefficient and the stability of this controller has been confirmed [11]. The structure of self-tuning fuzzy PID controller is shown in Fig. 4. 


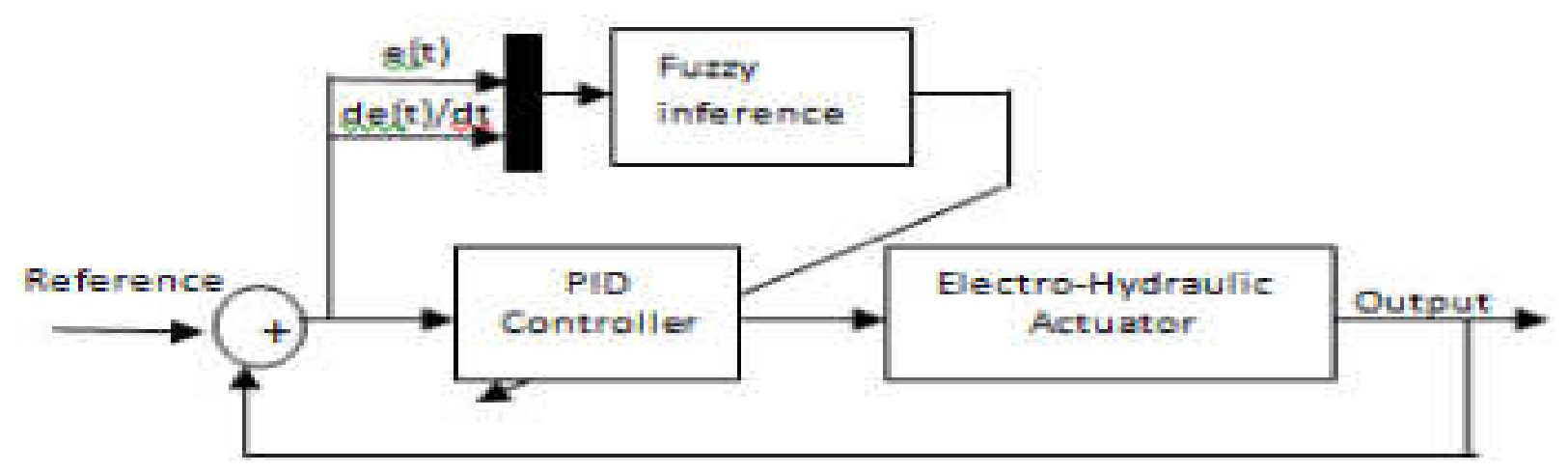

Fig.4. Fuzzy PID block diagram

The system consists of fuzzy interference block, PID controller block and the electro-hydraulic actuator block. For fuzzy logic controller, the feedback error e(t) and the derivative of error de(t)/dt as inputs which each of the input used five membership function. The outputs of the fuzzy logic controller are $K_{p}, K_{i}$, and $K_{d}$. Mamdani model is used as structure of fuzzy interference to obtain best value for $K_{p}, K_{i}$ and $K_{d}$.

There are two methods, Mamdani and Sugeno that are popular in fuzzy control design. The consequent of If-Then rule for Mamdani method explained by fuzzy set and the output is the reshaped by a matching number before defuzzification. On the contrary, consequent of If-Then rule for Sugeno method is defined by polynomial with respect to input variables, making the output of each rule a single number. Sugeno method does not involve defuzzification; however the step to determine the parameters of polynomials is not efficient and unclear than Mamdani [11-12]. Hence Mamdani interference is more popular in fuzzy control logic design. The fuzzy logic system is shown in Fig. 5.

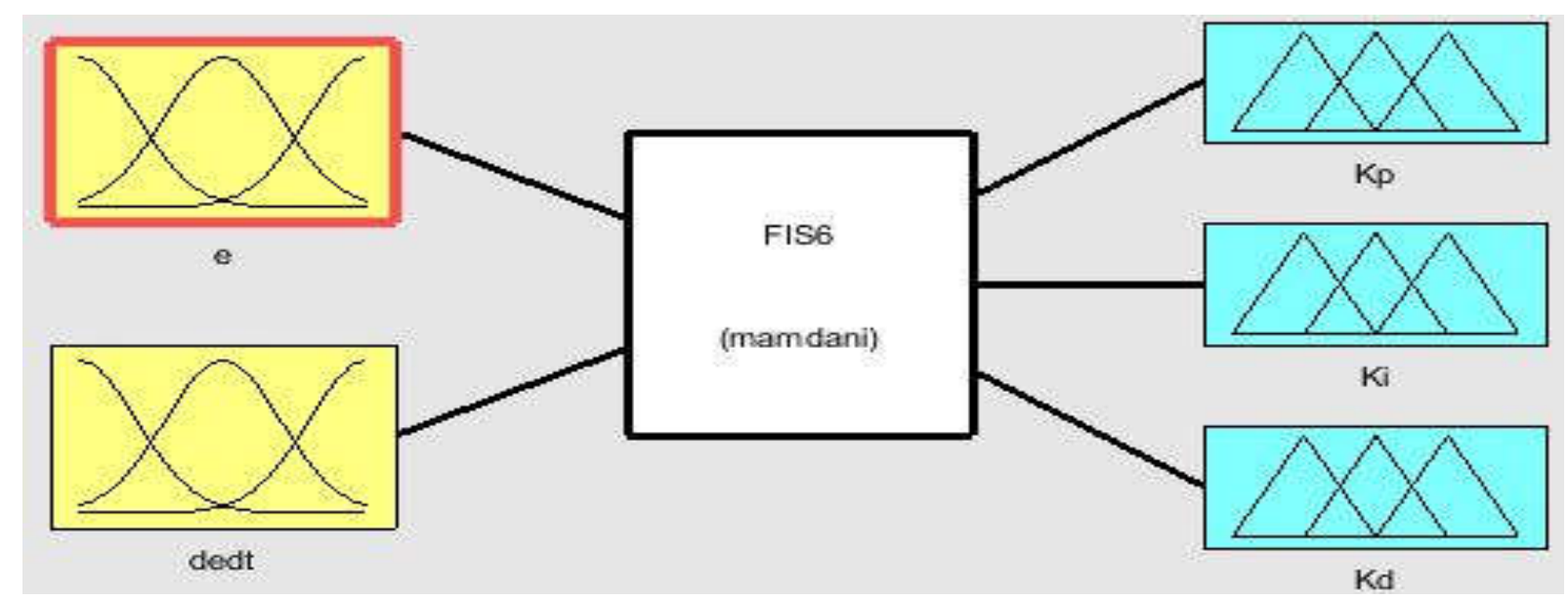

Fig.5. Fuzzy logic control system

All ranges of inputs and outputs were set during the conventional PID testing that has been 
conducted earlier. The values then substituted to the equation to compute the coefficients of $\mathrm{K}_{\mathrm{p}}, \mathrm{K}_{\mathrm{i}}$ and $\mathrm{K}_{\mathrm{d}}$ in Equation (4). It is important so that a feasible rule base with high frequency efficiency is obtained.

$$
\begin{aligned}
& K p=\frac{k p-k p_{\min }}{k p_{\max }-k p_{\min }} \\
& K i=\frac{k i-k i_{\min }}{k i_{\max }-k i_{\min }} \\
& K d=\frac{k d-k d_{\min }}{k d_{\max }-k d_{\min }}
\end{aligned}
$$
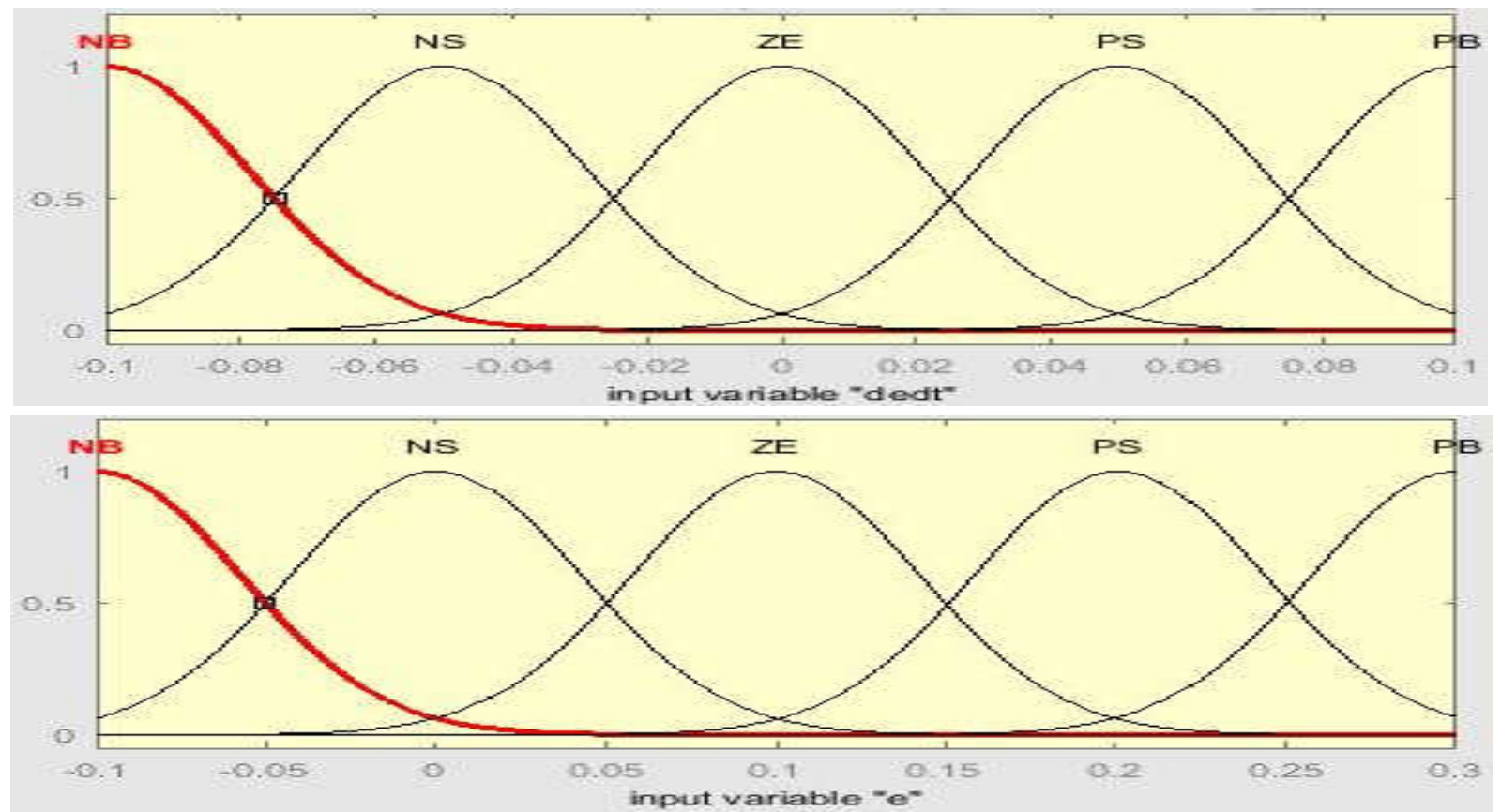

Fig.6. Input membership functions

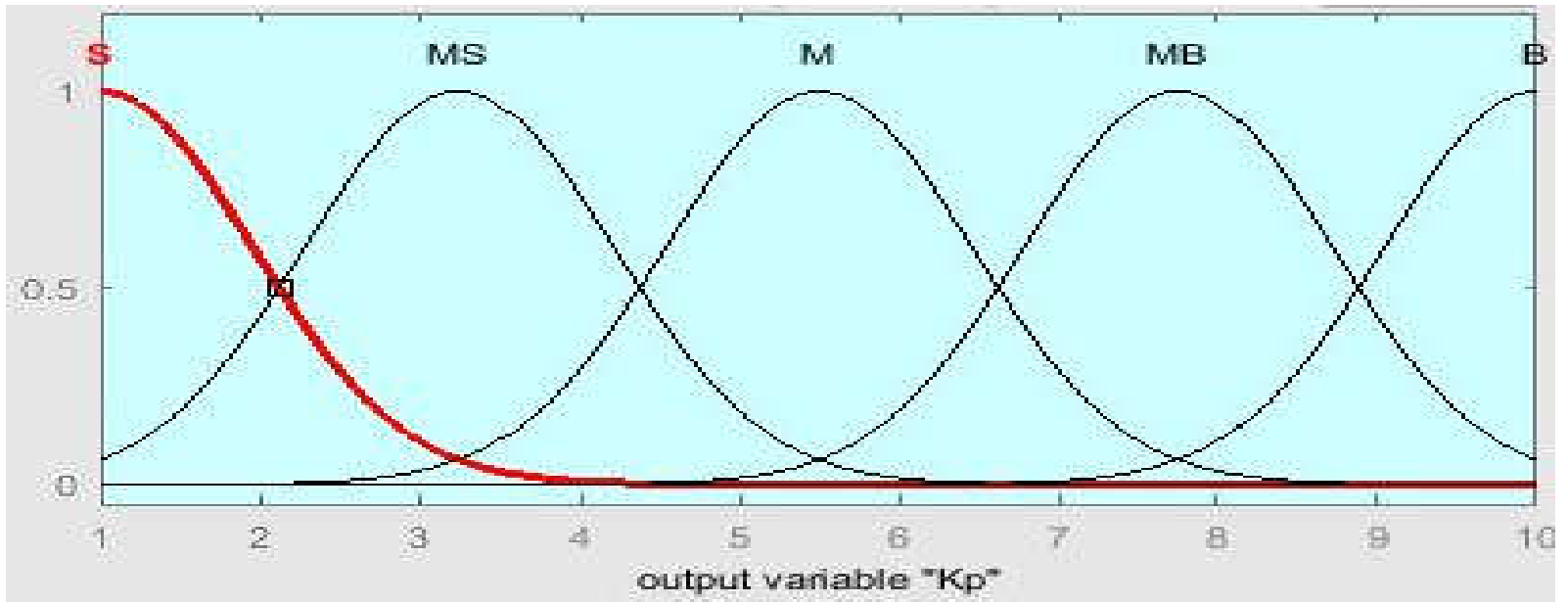




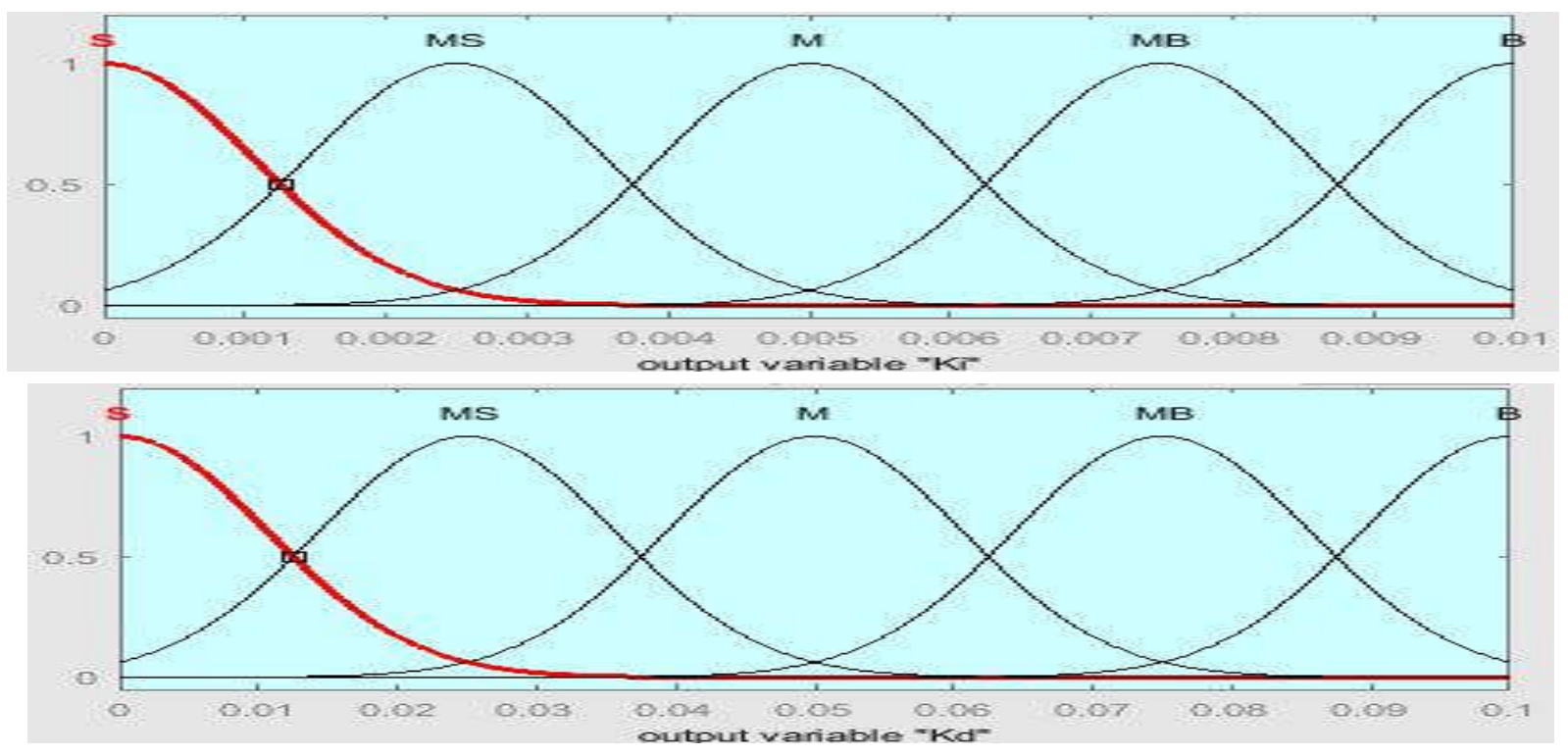

Fig.7. Output membership functions

The input membership function is shown in Fig. 6 and output membership function in Fig. 7. The fuzzy interference rule is shown in Table 2 and the linguistic variable levels are assigned as Small (S), Medium Small (MS), Medium (M), Medium big (MB) and Big (B). Hence, 25 fuzzy rules designed from the 5 linguistic variables.

Table 2. Fuzzy interference rule

\begin{tabular}{cccccc}
\hline De/dt & \multicolumn{5}{c}{ Error(e) } \\
& NB & NS & ZE & PS & PB \\
\hline NB & S & S & MS & MS & M \\
NS & S & MS & MS & M & MB \\
ZE & MS & MS & M & MB & MB \\
PS & MS & M & MB & MB & B \\
PB & M & MB & MB & B & B \\
\hline
\end{tabular}

\section{RESULTS AND DISCUSSION}

This section discussed about the simulation results analysis to show the effectiveness of the designed controller. The simulated output signal for different sampling times with step input signal and square input signal are shown in Figure 8 and Figure 9. The shape is chosen such that to demonstrate the ability of the controller to track the trajectory with changing frequency component. 


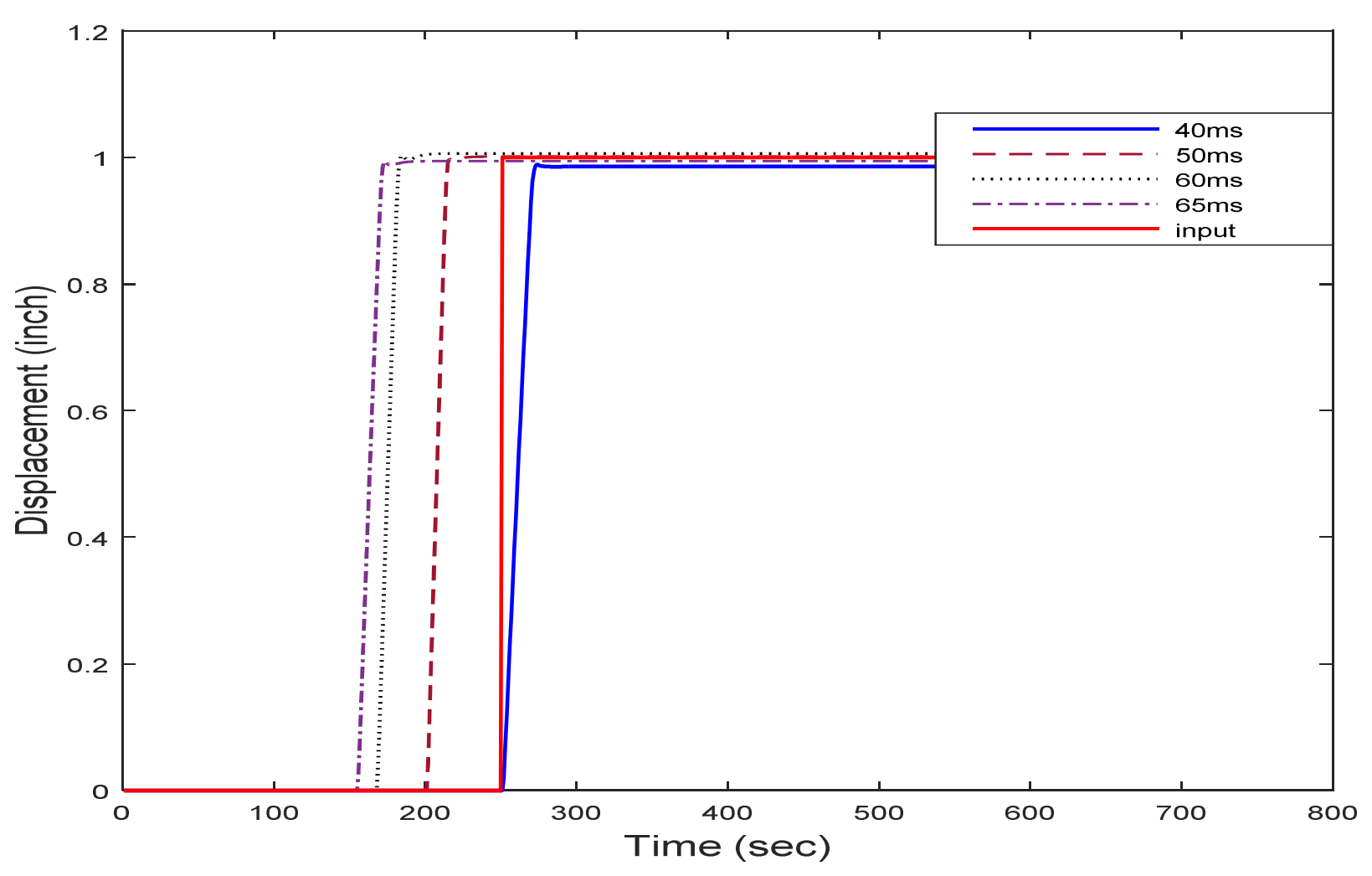

Fig.8. (a) Simulated output using step input

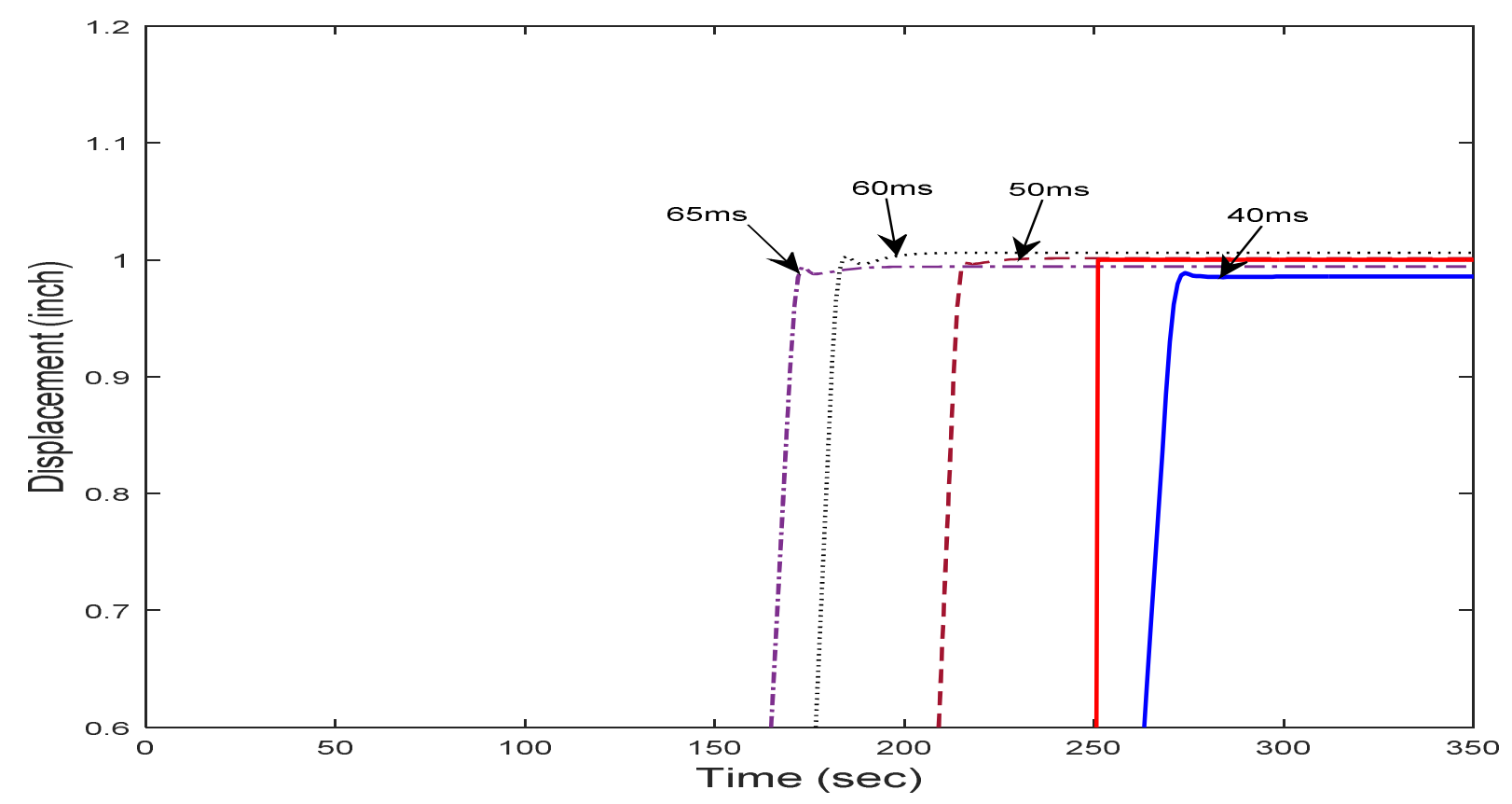

Fig.8. (b) Magnified simulated output using step input

The simulation results using step input to the control system are given in Fig. 8. The graph is zoomed at the sharp angles to observe the performance differences. From Fig. 8(b), all models show the transient response is a bit slower but model 50ms no overshoot. Other models show faster response but with overshoot. 


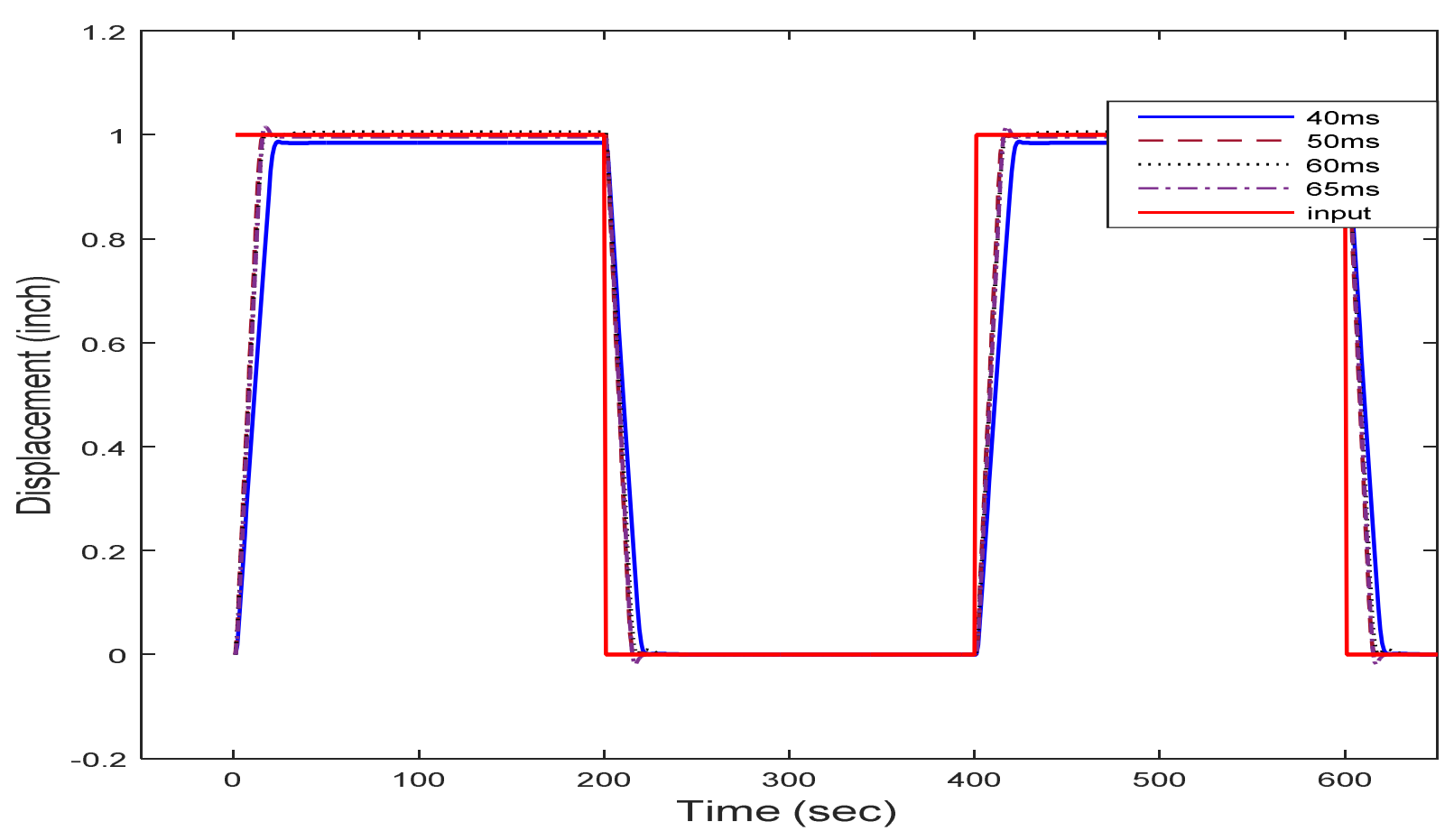

Fig.9. (a) Simulated output using square input



Fig.9. (b) Magnified simulated output using square input

The simulation results using square-wave input to the control system are given in Fig. 9. The graph is also zoomed at the sharp angles to observe the performance differences. From Fig. 9(b), model 50ms show the transient response is a bit slower but no overshoot. Other model show faster response but with overshoot. 


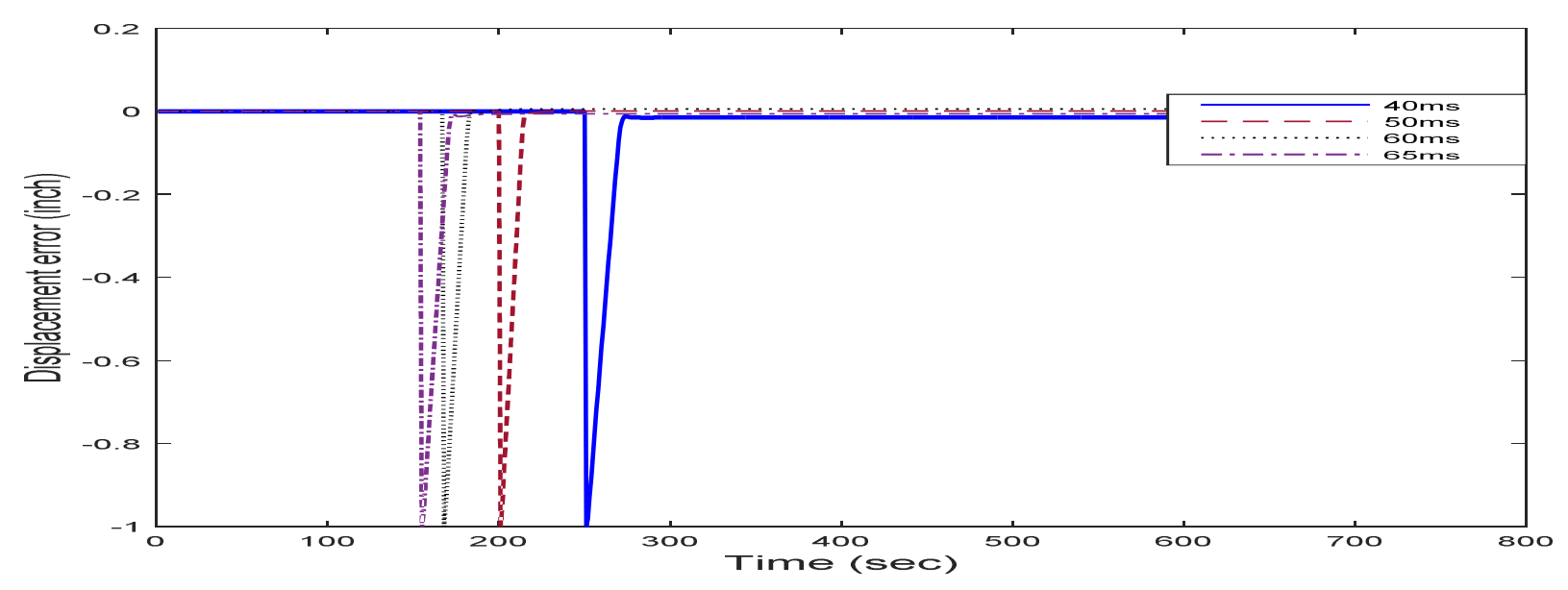

Fig.10. Tracking error using step input

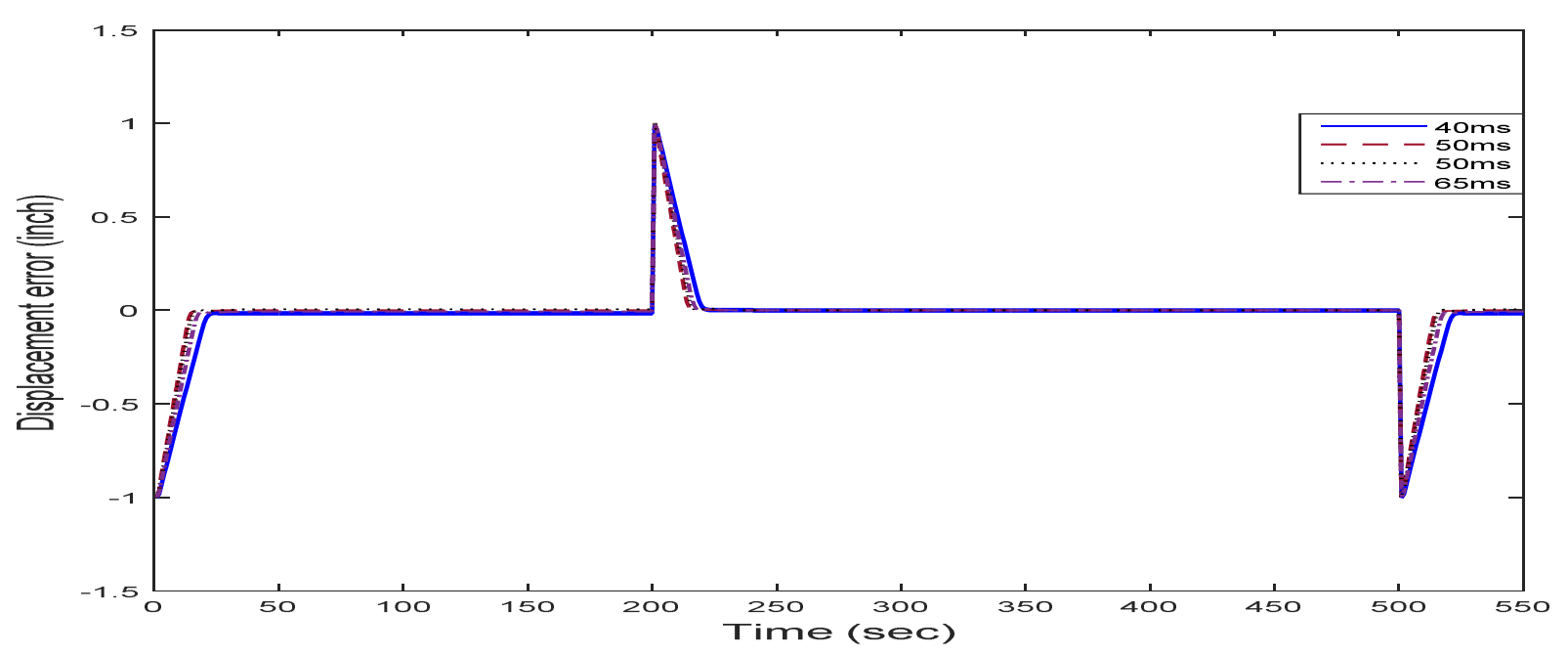

Fig.11. Tracking error using square input

Root Mean Square Error (RMSE) for both inputs was calculated to compare which model is more consistent. Based on the modeling conducted earlier, $40 \mathrm{~ms}$ model has zero located outside and further from unity circle, results in slightly higher RMSE. Zero location for 50ms model also outside but closer to the unity circle, thus produce smaller RMSE value. $60 \mathrm{~ms}$ and $65 \mathrm{~ms}$ model have zero located inside the circle however they comes with higher RMSE value.

Table 3. Result of root mean square error

\begin{tabular}{ccc}
\hline Sampling & \multicolumn{2}{c}{ RMSE } \\
Time (m sec) & Step Input & Square Input \\
\hline 40 & 0.0788 & 0.1862 \\
50 & 0.0716 & 0.1470 \\
60 & 0.0812 & 0.1637 \\
65 & 0.0900 & 0.1781 \\
\hline
\end{tabular}


From the simulation, the results showed that the model with 50ms sampling times gave better performance.

\section{CONCLUSION}

The model of the plant was obtained using model identification method through MATLAB System Identification Toolbox. Fuzzy PID controller was designed and applied to the system in order to test the performance of the model. The model was then compared with four different sampling times. The root mean square of tracking errors from the simulated shows impressive results that mean that the control strategy can be implemented practically.

\section{ACKNOWLEDGEMENTS}

This work was conducted at the Faculty of Electrical Engineering, UiTM facilities with financial support from 600-IRMI/MYRA 5/3/LESTARI (0001/2016). The authors would like to thank to the Institute of Research Management and Innovation (IRMI) and UiTM for their support.

\section{REFERENCES}

[1] Cologni A L, Mazzoleni M, Previdi F. Modeling and identification of an electro-hydraulic actuator. In 12th IEEE International Conference on Control and Automation, 2016, pp. 335-340

[2] Ling T G, Rahmat M F, Husain A R. System identification and control of an Electro-Hydraulic Actuator system. In IEEE 8th International Colloquium on Signal Processing and its Applications, 2012, pp. 85-88

[3] Ling $T$ G, Rahmat M F, Husain A R, Ghazali R. System identification of electro-hydraulic actuator servo system. In 4th IEEE International Conference On Mechatronics, 2011, pp. 1-7

[4] Izzuddin N H, Johari M R, Osman K. System identification and predictive functional control for electro-hydraulic actuator system. In IEEE International Symposium on Robotics and Intelligent Sensors, 2015, pp. 138-143 
[5] Carvajal J, Chen G, Ogmen H. Fuzzy PID controller: Design, performance evaluation, and stability analysis. Information Sciences, 2000, 123(3):249-270

[6] Adnan R, Tajjudin M, Ishak N, Ismail H, Rahiman M H. Self-tuning fuzzy PID controller for electro-hydraulic cylinder. In IEEE 7th international colloquium on Signal Processing and its Applications, 2011, pp. 395-398

[7] Kandiban R, Arulmozhiyal R. Speed control of BLDC motor using adaptive fuzzy PID controller. Procedia Engineering, 2012, 38:306-313

[8] Amer A F, Sallam E A, Elawady W M. Fuzzy pre-compensated fuzzy self-tuning fuzzy PID controller of 3 DOF planar robot manipulators. In IEEE/ASME International Conference on Advanced Intelligent Mechatronics, 2010, pp. 599-604

[9] Liem D T, Truong D Q, Ahn K K. A torque estimator using online tuning grey fuzzy PID for applications to torque-sensorless control of DC motors. Mechatronics, 2015, 26:45-63

[10] Taib M. N., Ramli A., Mohd H. F. R. Practical system identification. Selangor: Universiti Teknologi MARA Press, 2007

[11] Wang C. A study of membership functions on mamdani-type fuzzy inference system for industrial decision-making. Master thesis, Pennsylvania: Lehigh University, 2015

[12] Sebastião A, Lucena C, Palma L, Cardoso A, Gil P. Optimal tuning of scaling factors and membership functions for mamdani type PID fuzzy controllers. In IEEE International Conference on Control, Automation and Robotics, 2015, pp. 92-96

\section{How to cite this article:}

Ishak N, Hamdan NS, Tajuddin M, Adnan R. Modeling and controller design on ARX model of electro-hydraulic actuator. J. Fundam. Appl. Sci., 2017, 9(4S), 160-172. 